\title{
Recognition of Osteoporosis through CT-Images
}

\author{
Mahantesh Elemmi ${ }^{1}$, Gurusiddappa Hugar ${ }^{2}$, Shanta Kallur ${ }^{3}$ \\ Assistant Professor, Computer Science, KLEIT, Hubli, INDIA ${ }^{1,2,3}$
}

\begin{abstract}
Image processing has a very big potential to do virtually anything. This project comes to the extent of development details on Recognition of osteoporosis through CT images. The objective of the recognition of osteoporosis is to identify and distinguish between a normal bone image and osteoporotic bone image with its case as severe or non-severe. Osteoporosis is due to the following two phenomena: a reduction in bone mass and a degradation of the micro architecture of bone tissue. Osteoporosis is a disease in which the quality of bone is reduced, leading to weakness of the skeleton and increased risk of fracture and change is observed in micro architecture. In this project we propose a methodology to build a system to identify the normal bone image and affected bone image with the case severe or non severe. We use contrast feature of the grey level co-occurrence matrix and apply thresholding to detect the normal or osteoporotic bone image.
\end{abstract}

Keywords: Osteoporosis, Computer Vision, Bone Images, Endocrine, Rule Based Classification

\section{INTRODUCTION}

Computer vision is a field that includes methods for acquiring, processing, analysing, and understanding images and in general, high-dimensional data from the real world in order to produce numerical or symbolic information. A theme in the development of this field has been to duplicate the abilities of human vision by electronically perceiving and understanding an image. As a scientific discipline, computer vision is concerned with the theory behind artificial systems that extract information from images. The image data can take many forms, such as video sequences, views from multiple cameras, or multi-dimensional data from a medical scanner. Computer vision and image processing are widely used in industry, biological science, material science, medical science, weather forecasting and many other fields.

In our system, computer vision is being applied to medical field. Medical field is an aggregation of sectors within the economic system that provides goods and services to treat patients with curative, preventive, rehabilitative, and palliative care. The medical field is divided into many sectors to meet health needs of individuals and populations. The health care industry is one of the world's largest and fastest-growing industries. Medical industry forms an enormous part of a country's economy.

In the system we are concerned on a part of orthopaedic related disease known as osteoporosis. The system is inspired for the poor people who are not capable to go for high-end testings carried out for finding the osteoporosis. It is designed for the ease of use for the people to know about their level of disease with less time consumption and minimum cost.

\section{Proposed System}

The broad classification of the normal and affected bone images according to the age groups are shown in figure 1 .

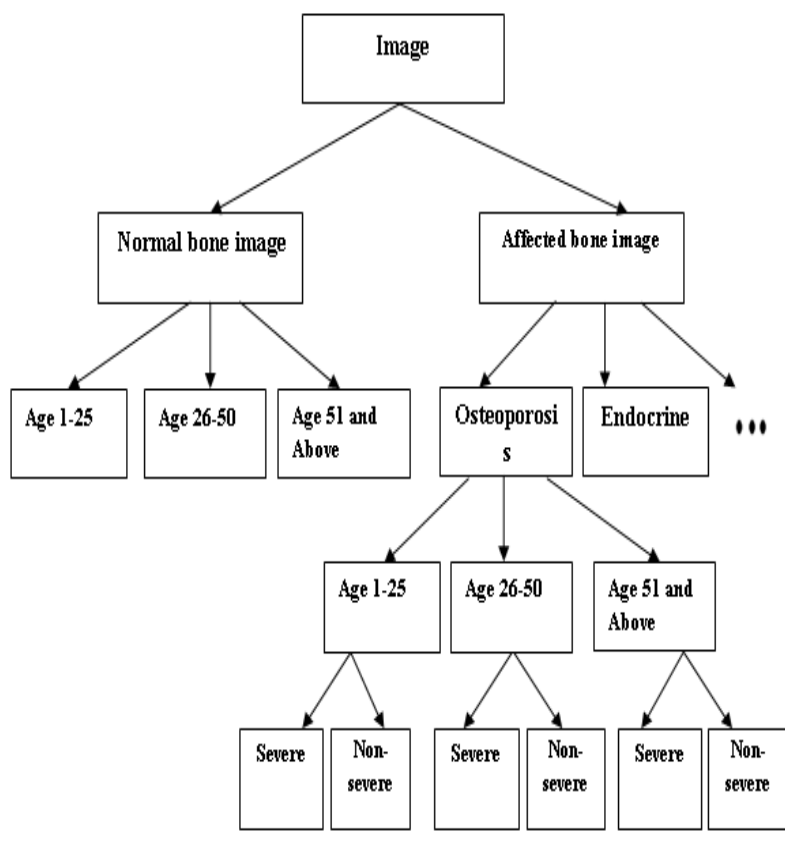

Fig.1: Broad classification of proposed work

The bone images are classified into normal bone images and affected bone images. The difference between the normal bone image and affected bone image is identified by checking the thickness between the cortical bone and cancellous bone. The normal bone image consists of more number of white pixels and an affected bone image consists of more number of black pixels. The normal bone images are further categorized into three age groups that is the age group of 1 to 25 , age group of 26 to 50 and age group of 51 and above. The categorized age group images are further classified into severe and non-severe osteoporotic bone images. The normal bone images categorised into the different age groups from 1 to 25,26 to 50 and 51 and above are as shown in the figure 2 . 
Normal bone images
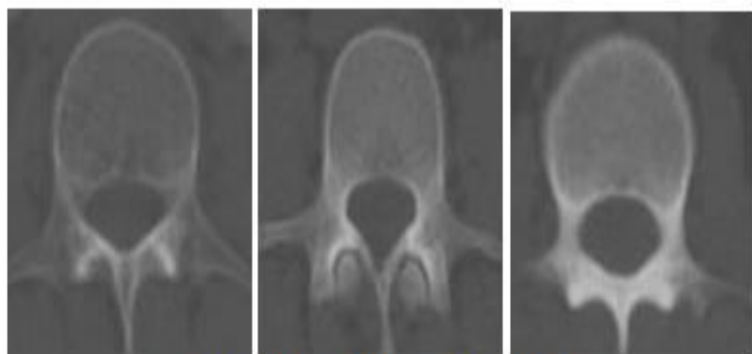

Normal Bone images of age group 1.25
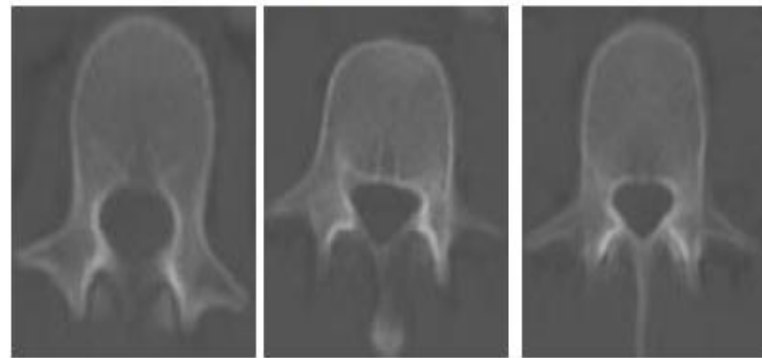

Normal Bone images of age group $26-50$
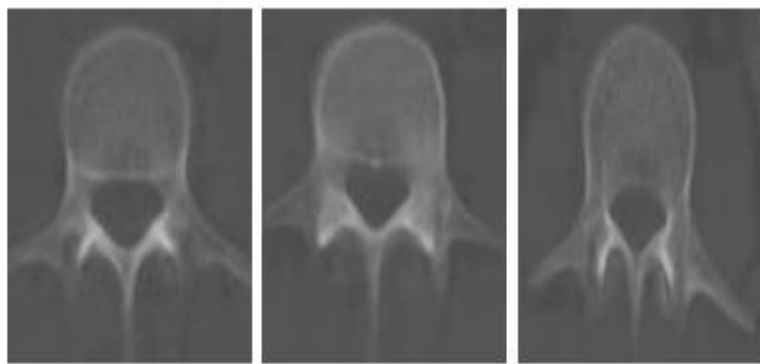

Normal B one im ages of age group 51 and A bove
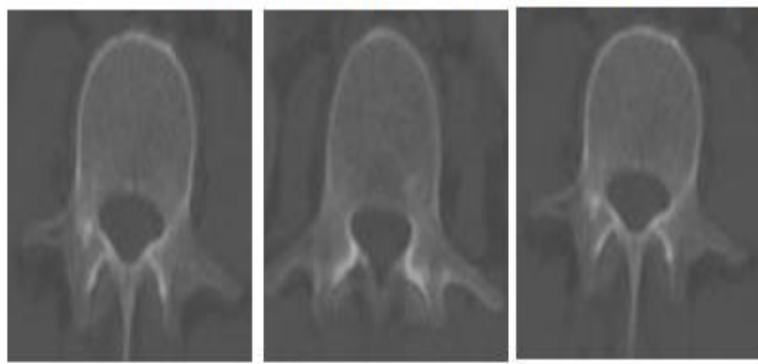

Fig.2. Normal bone images

In the affected bone images, we are dealing with the disease osteoporosis. If the thickness between the cortical and cancellous bone decreases then the calcium content in the bone is said to be reduced. And an osteoporotic bone image consists of more number of black pixels.

The affected bone images are categorised into the different age groups from 1 to 25,26 to 50 and 51 and above are as shown in the figure 3 .
Osteoporotic bone images
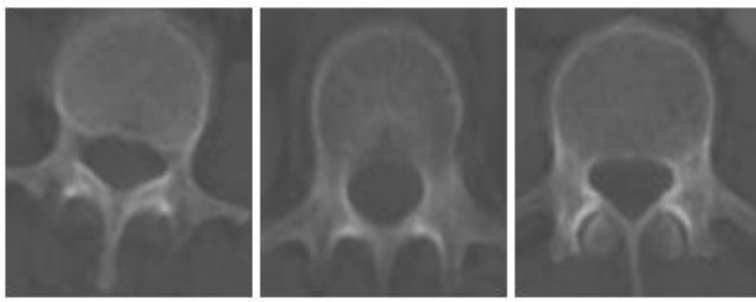

A ffected B one images of age group $1-25$

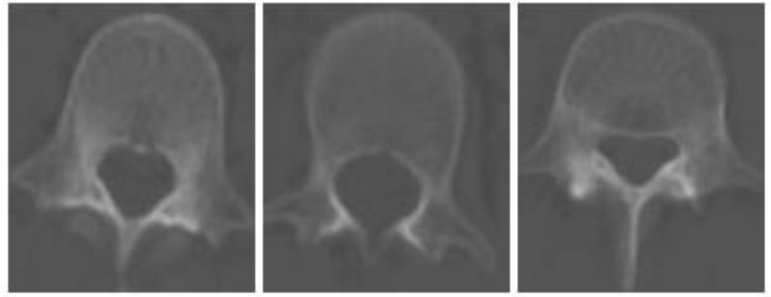

A ffected B one images of age group $26-50$
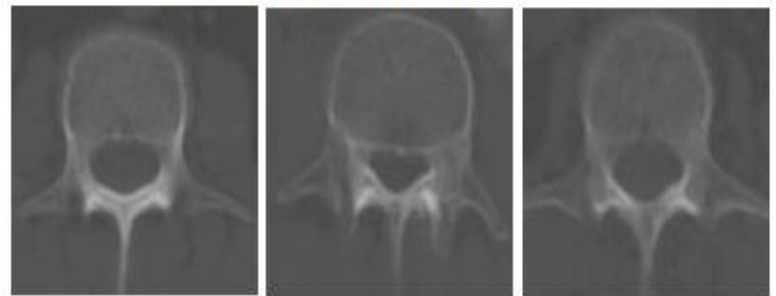

Affected $B$ one images of age group 51 and $A$ bove
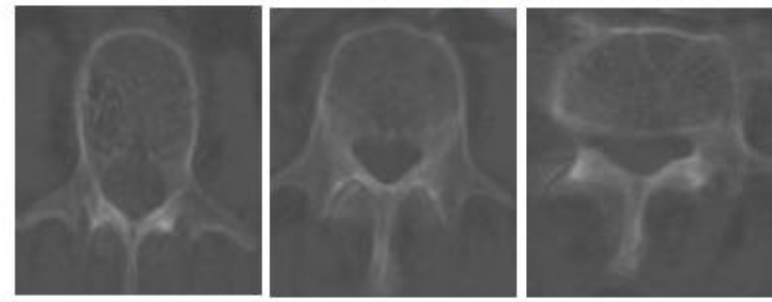

Fig.3. Affected bone images

Depending on the thinness of the bone the severity of the bone image is detected. The severity and non-severity of the osteoporotic bone images of each age group are detected. The samples of severe osteoporotic bone images and non-severe osteoporotic bone images are shown in the figure. 4 .

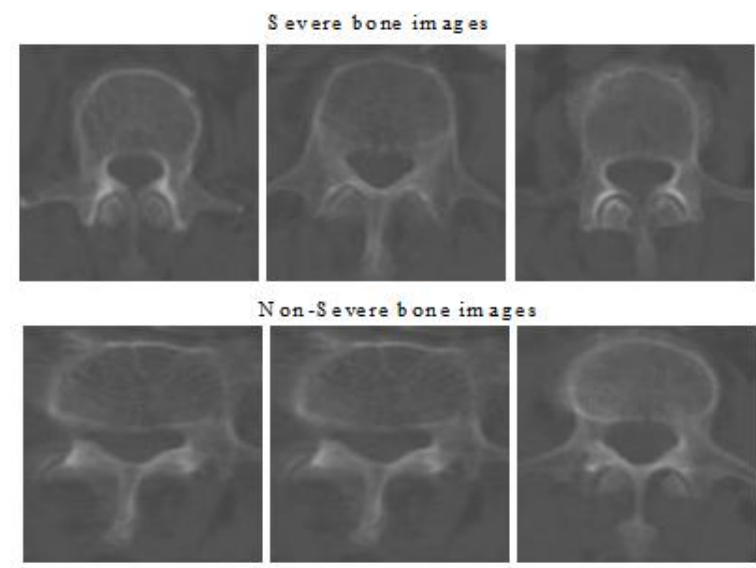

Fig.4. Osteoporotic images based on severity 


\section{III.DESIGN \& IMPLEMENTATION}

The design phases of the proposed work are as shown in the figure 5. The phases consist of image acquisition, preprocessing, image segmentation, feature extraction and rule based classification.

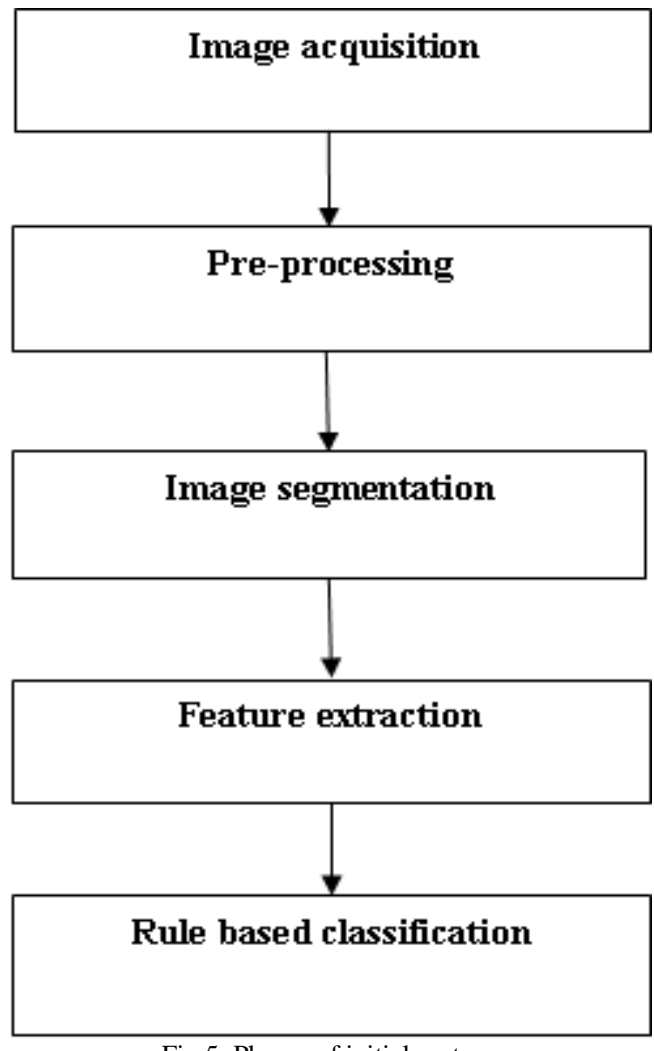

Fig.5. Phases of initial system

Image acquisition: $\mathrm{CT}$-images are acquired from various hospitals by visiting the hospitals frequently. Images are classified into normal bone images and affected bone images. The images are acquired on the basis of factors like age and gender. The information related to the patients such as the patient's name, age and gender are not revealed in the images we are using for training our system. After the frequent visits to the hospital the total numbers of images obtained are 192 bone images. Out of the 192 images acquired, 78 are the normal bone images and 114 are the affected bone images. According to images we collected we get to know that the osteoporotic disease occurs more for the older age people rather than the young age people. The samples our data collections are shown in the figure 6 . And in the figure 7 .

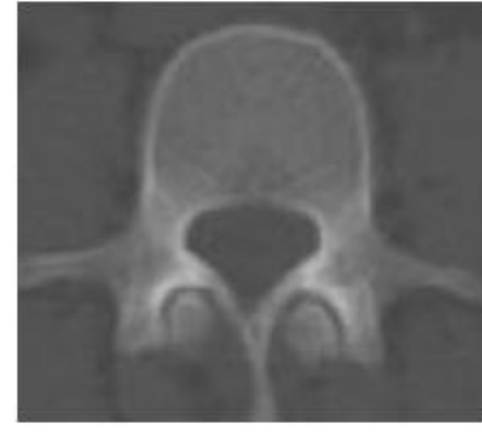

Fig.6. Normal bone image
Pre-processing: Usually the images that are obtained during image data collection may not be suitable for classification purpose because of certain factors, such as lighting intensity and size variations and some noise introduced by devices. We carried out the following work in pre-processing.

Resize: The acquired images are resized into a specific dimension.

Crop: The part of the image is cropped as needed.

Image segmentation: In image segmentation the required part is obtained by segmenting CT images. Grey level thresholding is being used. It is a fundamental tool for segmentation of grey level images when objects and background pixels can be distinguished by their grey level values. After cropping and resizing of the grey level image we apply grey level thresholding. By applying thresholding we identified the difference between the normal and affected bone images by calculating the total number of white and black pixels.

If the white pixels are more in number and black pixels are lesser in number, in an image then it is considered to be a normal bone image. If the black pixels are more in number and white pixels are lesser in number, in an image then it is considered to be affected bone image. The bone images after segmentation are shown in the figure 8 .
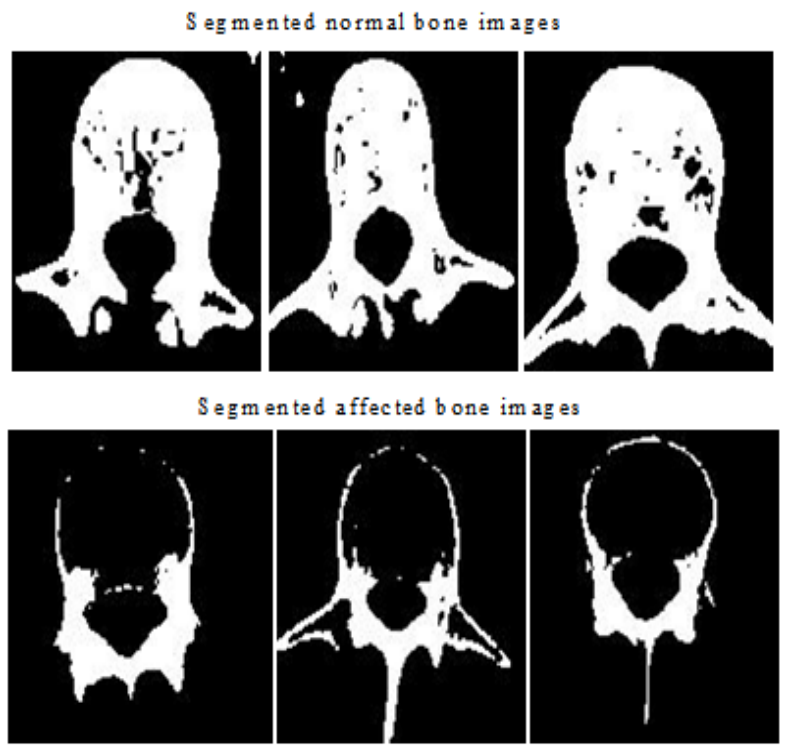

Fig.8. Segmented images 
Feature extraction: The Gray level co-occurrence matrix (GLCM) features are extracted. Among entropy, contrast, energy, homogeneity and correlation features of GLCM the contrast feature values gives the accurate result. The mean and standard deviation values are calculated for the contrast feature values. The mean function used is

$$
\bar{x}=\frac{1}{n} \sum_{i=1}^{n} x_{i}
$$

And for calculating the standard deviation the function used is

$$
s=\left(\frac{1}{n-1} \sum_{i=1}^{n}\left(x_{i}-x^{-}\right)\right)^{\frac{1}{2}}
$$

The input image's contrast values are then compared with the obtained mean and standard deviation values and the result is displayed as whether the given input image is normal bone image and affected bone image. If the image is affected bone image then it is further identified as whether it is severe bone image or non-severe bone image.

The contrast values for different type of bone images can be observed from the tables below. The mean values for affected bone images are shown in table 1 . The standard deviation values for affected bone images are shown in table 2. The mean values for normal bone images are shown in table 3 . The standard deviation values for normal bone images are shown in table 4 .

The mean and standard deviation values of the contrast feature of the normal and affected bone images are giving the accurate results when compared to other features such as correlation, entropy and homogeneity. From the tables we can observe that contrast values provide more accurate result than other feature values.

Table 1: Mean values for affected bone images

\begin{tabular}{|c|c|c|c|c|}
\hline Peatures & Contrast & Correlation & Entropy & Homogeneity \\
\hline 1 to 25 & 0.0554 & 0.9240 & 0.4502 & 0.9722 \\
\hline 26 to50 & 0.0565 & & & 0.9721 \\
\hline 51 and Above & & 0.9079 & 0.5092 & \\
& 0.0519 & 0.8867 & 0.5614 & 0.97405 \\
\hline
\end{tabular}

\section{IV.RESULTS}

Step 1: Store the collected normal and affected CT images for training in the database.

Step 2: Extract the Gray Level Co-occurrence matrix features which includes homogeneity, correlation, entropy, contrast and energy. The contrast values give the accurate result store the contrast feature value in the database.

Classification: The classifier used for our system is Rule approach. Initially in the classification phase we that is whether it is osteoporotic or not. If the bone is osteoporotic we then find whether the disease is severe or non-severe.

Table 3: Mean values for normal images

\begin{tabular}{|c|ccc|c|}
\hline Peatures & Contrast & Correlation & Entropy & Homogeneity \\
\hline 1 to 25 & & & \\
& & & \\
26 to 50 & 0.0417 & 0.9345 & 0.4688 & 0.9791 \\
& 0.0434 & 0.9282 & 0.5006 & 0.9782 \\
\hline 51 and Above & & & & \\
& 0.0399 & 0.9255 & 0.5179 & 0.9800 \\
\hline
\end{tabular}

Table 4: Standard deviation values for normal images

\begin{tabular}{|c|c|c|c|c|}
\hline Centures & Conltrast & Correlation & Enltopy & Homogeneily \\
\hline 1 to 25 & & & & \\
\hline 26 to 50 & 0.007 & 0.0103 & 0.0282 & 0.0035 \\
\hline 51 and $A$ bove & 0.0073 & 0.0144 & 0.0315 & 0.0036 \\
& 0.0049 & 0.0104 & 0.0181 & 0.0024 \\
\hline
\end{tabular}


Step 3: Calculate the number of white pixels of the images. Calculate the mean and standard deviation of white pixels and store it in database.

Step 4: Acquire an image from the user and crop obtained image and resize the image into [128X 128] dimension for making the image into a standard form of image for further computation.

Step 4: Extract the contrast feature from the obtained image from the user.

Step 5: Calculate the number of white pixels of the obtained image from the user.

Step 6: Calculate the number of white pixels of the obtained image from the user, calculate mean and standard deviation of white pixels and store it in database.

Step 7: Map the obtained result to training result that contains and display whether the image is osteoporotic and normal and if the image is osteoporotic then display whether the image case is severe or non-severe.

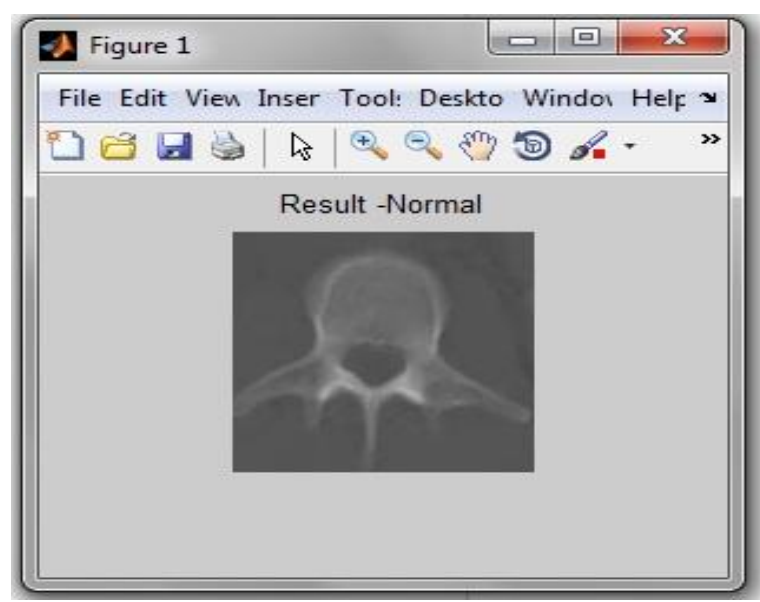

Fig.9. Normal bone image detected

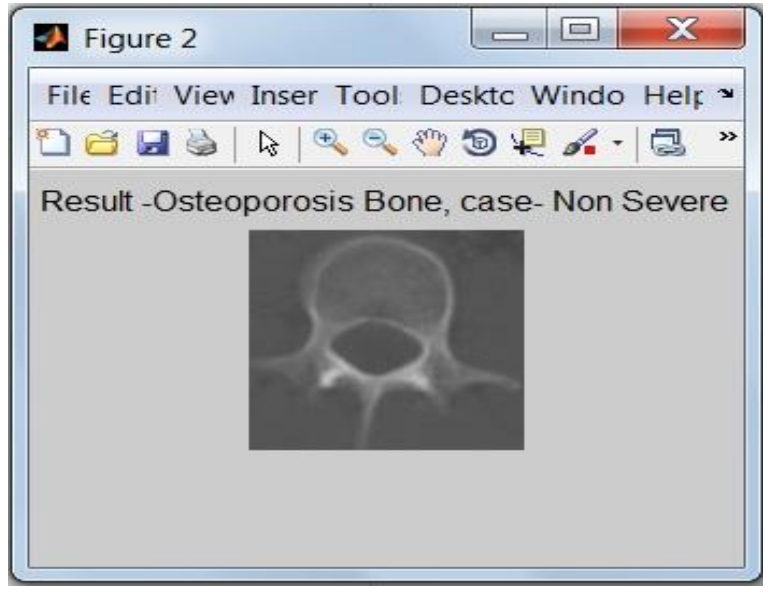

Fig.10. Non- severe osteoporotic bone image detected

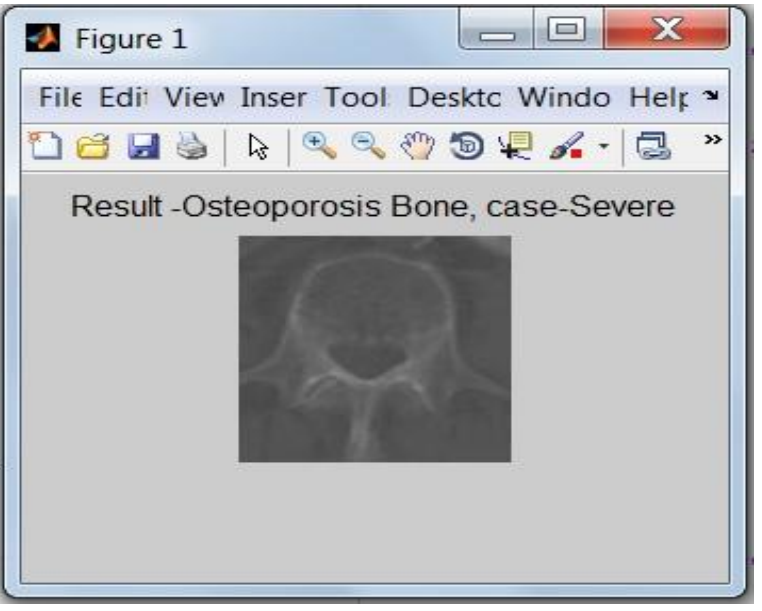

Fig.11. Severe osteoporotic bone image detected

Out of 192 bone images 78 are normal bone images and 114 are affected bone images. The recognition rate we obtained for normal bone images is $94.87 \%$ and for affected bone images is $92.10 \%$.

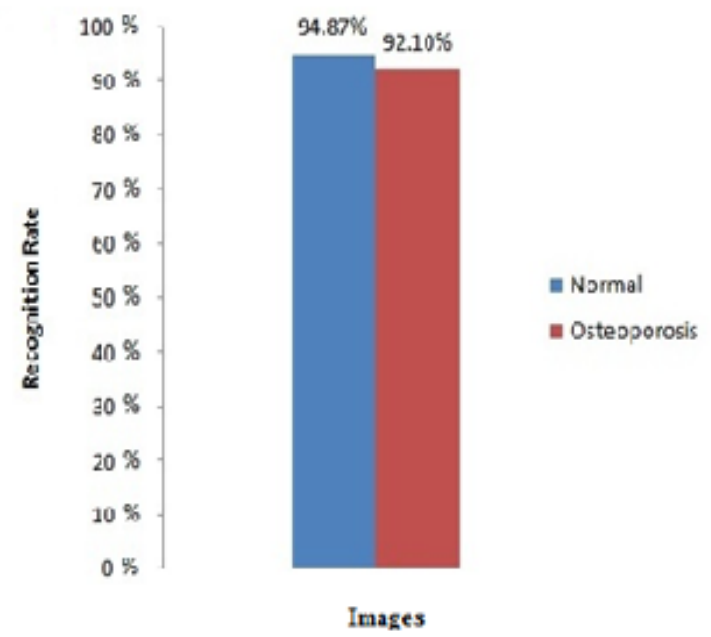

Fig.12. Total number of images detected

The overall recognition rate obtained for normal and osteoporotic bone images is $93.48 \%$. The system has been trained to distinguish between normal and affected, a confusion matrix will summarize the results of testing the algorithm for further inspection. Among 192 images 78 normal and 114 affected images the resulting confusion matrix could look like the table below:

Table 5: Confusion matrix

\begin{tabular}{|c|c|c|}
\hline & Normal & Affected \\
\hline Normal & 74 & 4 \\
\hline Affected & 7 & 107 \\
& & \\
\hline
\end{tabular}

Based on age factor the bone images are classified into major 3 sections. They are 1-25, 26-50, 51 and above age 
groups. The recognition rate for the age group 1 to 25 of The osteoporotic bone images are further classified into normal bone images is $96.66 \%$ and affected bone images severe bone images and non-severe bone images. The is $100 \%$. The recognition rate for the age group 26 to 50 of recognition rate for severe bone images is $95.65 \%$ and normal bone images is $95.23 \%$ and affected bone images non-severe bone images is $90.21 \%$.

is $96 \%$. The recognition rate for the age group 51 and above of normal bone an image is $100 \%$ and affected bone images are $95.44 \%$.

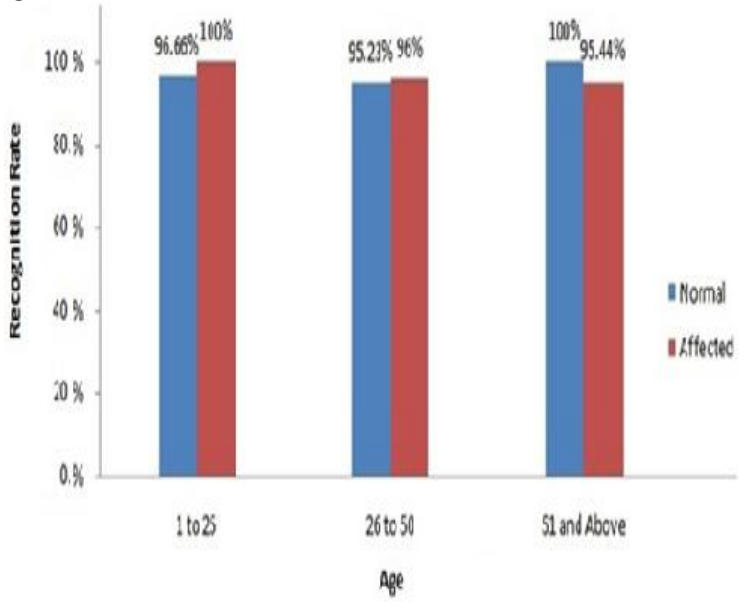

Fig.13. Comparison rate of images according to age.

The recognition rate obtained by extracting only the Gray Level Co-occurrence matrix feature for normal bone image is $58.97 \%$ and affected bone image is $93.85 \%$.

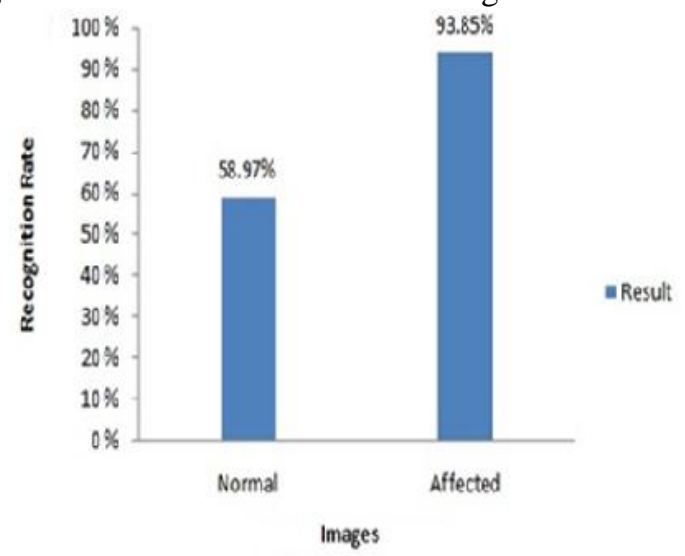

Fig.14. Recognition rate of images by extracting only GLCM features. The recognition rate obtained by extracting only the segmentation that is by plying Grey level thresholding for normal bone image is $98 \%$ and affected bone image is $79.82 \%$

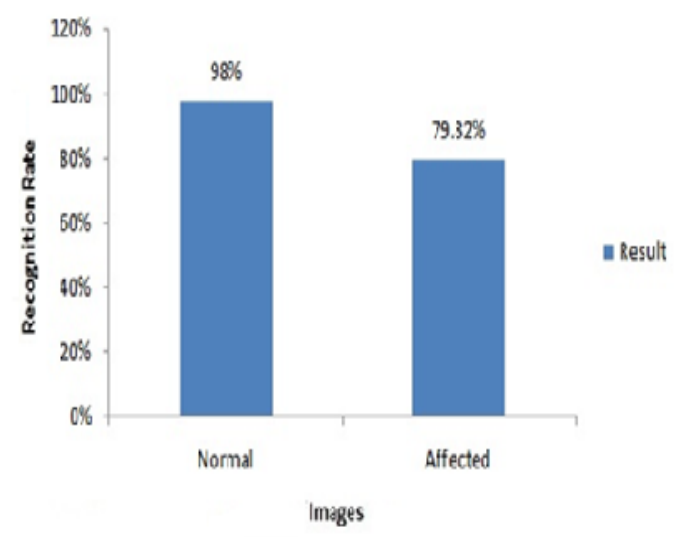

Fig.15. Recognition rate of images by segmentation

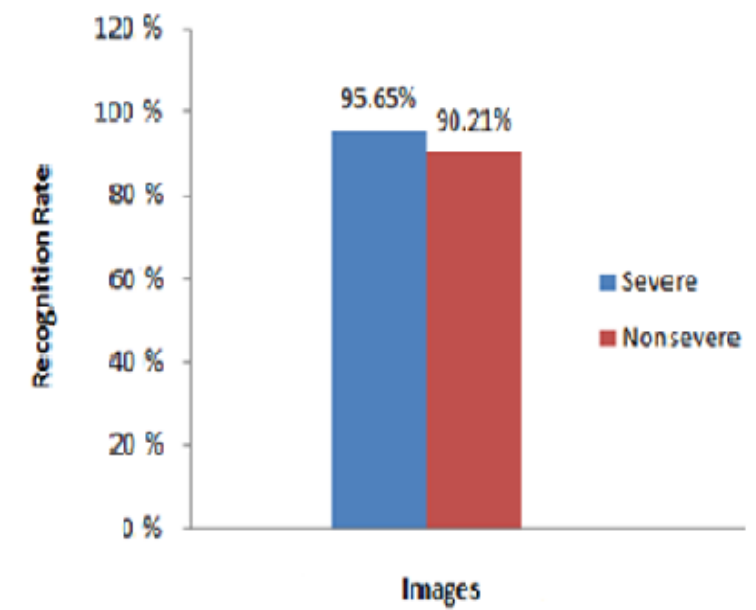

Fig.16. Recognition rate of osteoporotic images based on severity.

By considering $30 \%$ of training data and $70 \%$ of testing data, the obtained results are as follows. Out of 54 normal bone images 39 are detected and out of 81 affected bone images 64 are detected. The result in terms of percentage for normal bone images is $72.22 \%$ and affected bone images are $76.94 \%$.

Table 6: Recognition Rate by considering $30 \%$ of training data

\begin{tabular}{|c|c|c|}
\hline & Normal & Osteoporotic \\
\hline Detected & 39 & 64 \\
\hline Total & 54 & 81 \\
\hline Result & $72.22 \%$ & $76.94 \%$ \\
\hline
\end{tabular}

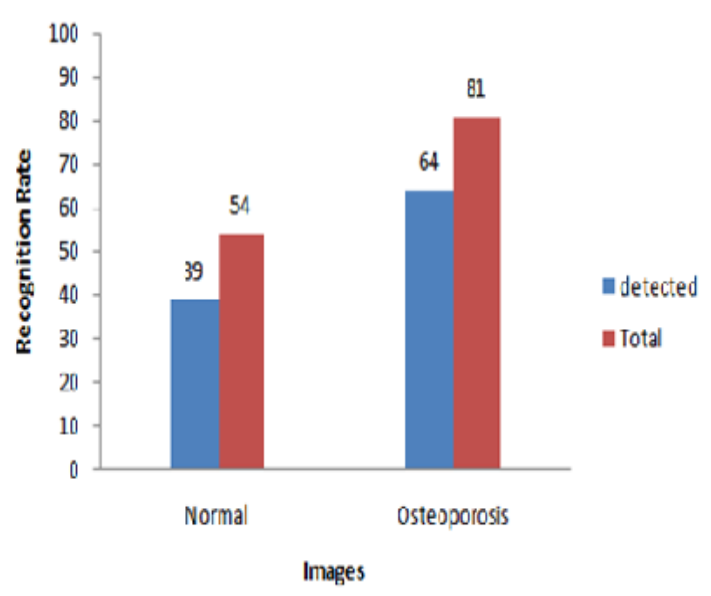

Fig.17. Recognition Rate by considering $30 \%$ of training data

By considering $50 \%$ of training data and $50 \%$ of testing data, the obtained results are as follows. Out of 38 normal 
bone images 34 are detected and out of 58 affected bone images 57 are detected. The result in terms of percentage for normal bone images is $90 \%$ and affected bone images are $98 \%$.

Table 7: Recognition Rate by considering $50 \%$ of training data

\begin{tabular}{|c|c|c|}
\hline & Normal & Osteoporotic \\
\hline Detected & 34 & 57 \\
\hline Total & 38 & 58 \\
\hline Result & $90 \%$ & \\
\hline & & \\
\hline
\end{tabular}

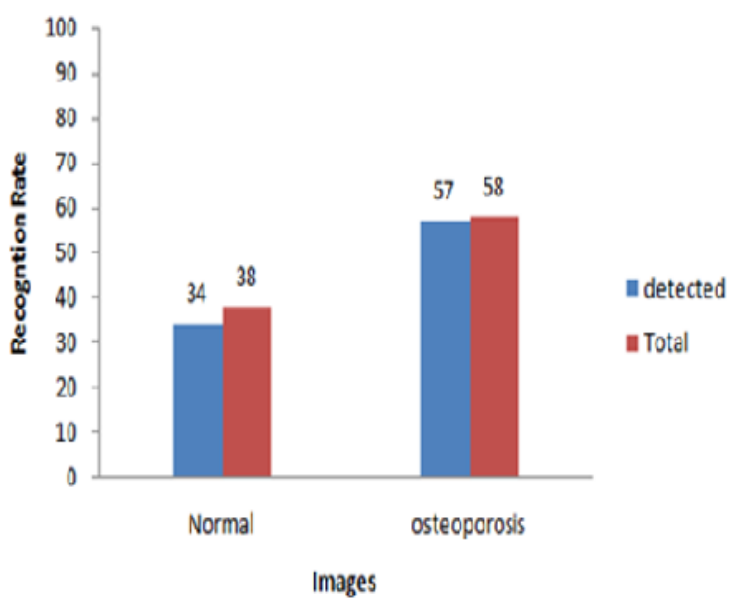

Fig.18. Recognition Rate by considering $50 \%$ of training data

By considering $70 \%$ of training data and $30 \%$ of testing data, the obtained results are as follows. Out of 25 normal bone images 24 are detected and out of 36 affected bone images 33 are detected. The result in terms of percentage for normal bone images is $96 \%$ and affected bone images are $92 \%$

Table 7: Recognition Rate by considering $70 \%$ of training data

\begin{tabular}{|c|c|c|}
\hline & Normal & Osteoporotic \\
\hline Detected & 24 & 33 \\
\hline Total & 25 & 36 \\
\hline Result & $96 \%$ & \\
\hline & & \\
\hline
\end{tabular}

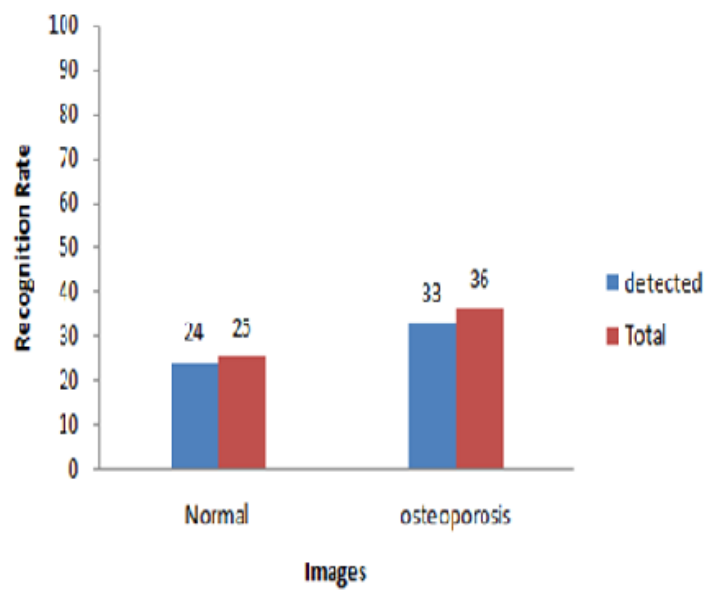

Fig.19. Recognition Rate by considering $70 \%$ of training data

By comparing $30 \%$ of training data, $50 \%$ of training data and $70 \%$ of training data we can say that only $50 \%$ of training data is sufficient to obtain good results.

\section{Conclusion}

In the past, several systems have been presented, which detects the osteoporosis on the parts of the body such as hip, arm and few systems were designed only for the classification of the diseases. There were less works carried out on the lumbar vertebrae bone. Thus, the development of faster classification methods and more accurate and precise features is very important in order to run such systems in real-time. This system classifies among the normal and abnormal images and detects the osteoporosis with the severity and non severity. Feature extraction is very important step in recognition of osteoporosis system. In this system feature extraction method is described. We have used Gray Level Cooccurrence matrix feature extraction method and segmentation. The experimental results show that the system has produced satisfactory recognition rate in finding about the osteoporosis occurred in lumbar vertebrae.

\section{ACKNOWLEDGMENT}

We are very grateful to our beloved Principal Dr. Basavaraj S. Anami, for providing all support and resources from the institution to complete the task in a stipulated time.

\section{REFERENCES}

[1] Liu ZQ, Austin T, Thomas CD, Clement JG. "Bone feature analysis using image processing techniques", Proceedings of the $20^{\text {th }}$ annual International conference of IEEE engineers in medicine and Biology society, Vol.20, No 2, 1998

[2] Abdurrahim Akgundogdu, Rachid Jennane, Gabriel Aufort, Claude Laurent Benhamou "3D image analysis and artificial intelligence for bone disease classification", Author manuscript, published in "Journal of Medical Systems 34, 5 (2010) 815-828" DOI : 10.1007/s10916-009-9296-3.

[3] Yi-King Choi, Leong Lu , Wenping Wang "VISBONE: 3D visualization of bone mineral density", Proceedings of the 29th Annual International Conference of the IEEE EMBS Cité Internationale, Lyon, France August 23-26, 2007.

[4] Humbert, Whitmarsh, De Craene, del Río Barquero "3D reconstruction of both shape and Bone Mineral Density distribution 
of the femur from DXA images", IEEE TRANSACTIONS ON MEDICAL IMAGING, VOL. 30, NO. 12, DECEMBER 2011

[5] Sooyeul Lee, Ji-Wook Jeong, Jeong Won Lee, Done-Sik Yoo “ Distal Radius Bone Mineral Density Estimation using the Filling Factor of Trabecular Bone in the X-ray Image", Proceedings of the 28th IEEE EMBS Annual International Conference, New York City, USA, Aug 30-Sept 3, 2006.

[6] Tristan Whitmarsh, Karl D. Fritscher, Ludovic Humbert, Luis M. Del-Rio-Barquero, Rainer Schubert andAlejandro F. Frangi “ Hip Fracture Discrimination using 3D Reconstructions from Dualenergy X-ray Absorptiometry", The 2012 Biomedical Engineering International Conference.

[7] Sahiti lahari M, Vijay, Anburajan M “ Finite Element Analysis of Femur in the Evaluation of Osteoporosis", Proceedings of the First Joint BMES/EMBS Conference serving Humanity, Advancing Technology Oct. 13-16, '99, Atlanta, GA, USA.

[8] Zhi Gao, Wenxue Hong, Yonghong Xu, Tao Zhang, Zhijie Song, Jian Liu "Osteoporosis Diagnosis Based on the Multifractal Spectrum Features of Micro-CT Images and C4.5 Decision Tree", 2010 First International Conference on Pervasive computing, signal processing and applications

[9] Yung-Yen Chiang, Shu-Li Wang, Shunghao Liu, Fan Wu " Using Cone Beam CT with Image Processing in Detecting the Bone Mineral Density of Jaw", $20114^{\text {th }}$ International Conference on Biomedical engineering and informatics.

[10] Sangeetha, S.; Dept. Of Instrum. Eng., Anna Univ., Chennai, India; Sujatha, C.M.; Manamalli, D; "Characterization of Trabecular Architecture in Femur Bone Radiographs Using Succolarity", in Vol. 202014

\section{BIOGRAPHIES}

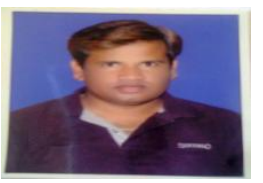

Mr. Mahantesh C. Elemmi Working as Asst.Professor in K.L.E. Institute of Technology Hubli, He received B.E. (CSE) \& M.Tech (CSE) from VTU Belgaum. His area of interest includes Image Processing, and all current trends and techniques in Computer Science.

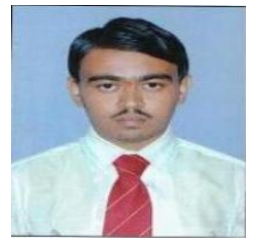

Mr. Gurusiddappa Hugar, Working as Asst.Professor in K.L.E. Institute of Technology Hubli; He received B.E. (CSE) \& M.Tech (DE) from VTU Belgaum. His area of interest includes Image Processing, Speech Processing, Web Technologies, all current trends and techniques in Computer Science.

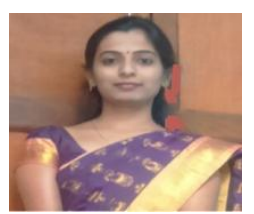

Ms. Shanta Kallur Working as Asst. Professor in K.L.E. Institute of Technology Hubli, She received B.E. (CSE) \& M.Tech (CSE) from VTU Belgaum. Her area of interest includes Image Processing, Computer Networks and all current trends and techniques in Computer Science. 